\title{
Optimal Multistage Schemes for Euler Equations with Residual Smoothing
}

\author{
Chang-Hsien Tai* and Jiann-Hwa Sheu ${ }^{\dagger}$ \\ Chung Cheng Institute of Technology, Tao-yuan, Taiwan 33509, Republic of China \\ and \\ Bram van Leer \\ University of Michigan, Ann Arbor, Michigan 48109-2140
}

\begin{abstract}
A numerical technique, composed of the Van Leer-Tai-Powell optimization and a modified procedure, is applied to design multistage schemes that give optimal damping of high frequencies for given upwind-biased spatial differencing with implicit and explicit residual smoothing. The analysis is done for a scalar convection equation in one space dimension. The object of this technique is to make the schemes suited for multigrid acceleration. The optimal multistage schemes and their damping properties are presented in this paper. By keeping the multistage coefficients from the one-dimensional analysis and simply redefining the Courant number, the schemes can be applied to multidimensional problems. A fast Euler code is used to evaluate the suitability of the schemes for multidimensional multigrid computations. Numerical results show that the modified schemes effectively enhance the convergence performance on both single and multiple grids.
\end{abstract}

\section{Introduction}

$\mathbf{T}$ HE computation of a steaty flow pattern induced by a specific fixed geometry has been done traditionally by implicit methods in the past few decades. This is due to the relatively large reduction in residual that can be achieved in one iteration step. Once multigrid relaxation was successfully implemented for solving hyperbolic equations, explicit methods became very effective and available for marching to steady-state solutions. Explicit methods have many advantages over implicit methods: they can be easily executed on computers with vector or parallel architectures, and they allow local grid refinement. The last advantage is crucial, since adaptive grid refinement seems to be the most promising way to efficiently obtain spatial accuracy in complex problems.

The explicit time-integration method proposed by Jameson et al. ${ }^{1}$ and Jameson, ${ }^{2}$ which is founded on separate time and space discretizations, is a remarkable combination of components including convergence-acceleration techniques and multigrid relaxation. Since the multigrid strategy acts to remove low-frequency components of the error while marching, it suffices for the singlegrid scheme used on each grid to damp only the high frequencies. $\mathrm{Tai}^{3}$ and Van Leer et al. ${ }^{4}$ have shown how to develop multistage integration methods that yield optimal damping of high-frequency modes. These schemes were derived for a scalar convection equation in one space dimension, and used to solve one- and multidimensional Euler equations. The analysis for optimizing the smoothing properties of multistage scheme was extended to a twodimensional scalar convection equation by Catalano and Deconick ${ }^{5}$ and, more satisfactorily, by Lynn and Van Leer. ${ }^{6}$ In the latter work, the optimization method is extended to the system of Euler equations, using the local preconditioning of Van Leer et al. ${ }^{7}$ to make the system act more like a scalar equation. These developments largely enhance the convergence performance of explicit methods.

The convergence-acceleration techniques introduced by Jameson $^{2}$ are local time-stepping, enthalpy damping, and residual smoothing. Among these techniques, implicit residual smoothing

Received Aug. 30, 1994; revision received Nov. 21, 1994; accepted for publication Dec. 20, 1994. Copyright (C) 1995 by the American Institute of Aeronautics and Astronautics, Inc. All rights reserved.

*Associate Professor, Department of Mechanical Engineering, Ta-shi.

${ }^{\dagger}$ Ph.D. Candidate, Department of System Engineering, Ta-shi.

${ }_{\ddagger}$ Professor, Department of Aerospace Engineering. Member AIAA.
(IRS) is particularly effective, since it increases the maximum allowable Courant number and smoothes the high-frequency component of the residual, as needed in multigrid relaxation. Jameson suggested some multistage schemes with good smoothing properties and derived a formula to obtain new Courant number while increasing smoothing coefficients. Enander ${ }^{8}$ also tried to improve the high-frequency damping properties of multistage schemes by using the developed combined implicit-explicit residual smoother. More recently, Blazek et al. ${ }^{9}$ introduced a upwind-biased operator for the IRS method and showed this method allows substantially higher Courant-Friedrichs-Lewy (CFL) numbers as compared to the centered IRS method. In this paper, only a central differencing operator for the implicit and explicit residual smoothing (ERS) is of interest.

In the present study we design optimally smoothing multistage schemes for given upwind-biased spatial discretizations ${ }^{10}$ using a central-difference IRS and ERS operator for extra smoothing and stability. They are designed by means of the Van Leer-TaiPowell optimization ${ }^{3,4}$ and a modified procedure. Since the spatial discretization dictates the final accuracy of the solution, the most natural way is to select the spatial discretization and then select the multistage coefficients in such a way that short waves are effectively damped. This means these schemes are designed purely for their properties of damping and stability, without regard to time accuracy. The search for the values of the parameters of the multistage schemes that yield optimal damping is done numerically.

Based on the finite volume formulation, ${ }^{2,11}$ a high-efficiency multigrid code for solving the Euler equations was programmed to simulate the axisymmetric external flowfield about a projectile. This code has been used for the actual multidimensional application of the optimal multistage schemes. Besides residual smoothing the code includes local time-stepping to speed up the rate of convergence and to make the smoothing analysis more appropriate. Convergence performance of these schemes and a comparison with the classical Runge-Kutta schemes are presented.

\section{Optimization of the Multistage Scheme}

\section{A. Van Leer-Tai-Powell Optimization}

Consider the linear ordinary differential equation

$$
\frac{\mathrm{d} u}{\mathrm{~d} t}=\lambda u, \quad \lambda \in C
$$


and use a predictor-corrector integration method to discretize the temporal term as

$$
\begin{gathered}
\tilde{u}=u^{n}+\alpha \lambda \Delta t u^{n} \\
u^{n+1}=u^{n}+\lambda \Delta t \tilde{u}=\left[1+\lambda \Delta t+\alpha(\lambda \Delta t)^{2}\right] u^{n}
\end{gathered}
$$

where the time-step ratio $\alpha$ is a free parameter. Apparently, the stability and damping properties are determined by the complex polynomial:

$$
P_{2}(z, \alpha)=1+z+\alpha z^{2}, \quad z=\lambda \Delta t
$$

This polynomial has two complex-conjugate zeros which move along a circle when varying $\alpha$.

When a partial differential equation is interpreted by the method of lines, $\lambda$ represents the Fourier transform of the spatial differencing operator and depends on the spatial frequency $\xi$ or, more specifically, on the spatial wave number $\beta=2 \pi \xi \Delta x$. For instance, consider the one-dimensional linear convection equation

$$
\frac{\partial u}{\partial t}=-c \frac{\partial u}{\partial x}, \quad c>0
$$

and discretize the space term by first-order upwind differencing. After inserting harmonic data $u(x)=u_{0} e^{-2 \pi i \xi x}$, Eq. (4) reduces to an equation of the form (1)

$$
\Delta t \frac{\partial u}{\partial t}=-\nu\left(1-e^{-i \beta}\right) u_{0} e^{-2 \pi i \xi x}
$$

where the nondimension time step $v=c \Delta t / \Delta x$ is the CFL number. All information about the spatial differencing operator is included in the complex function

$$
z(\beta)=-v\left(1-e^{-i \beta}\right)
$$

Similarly, the complex function for the kappa ${ }^{10}$ scheme becomes

$$
\begin{aligned}
& z(\beta)=-v\left(1-e^{-i \beta}\right)\left\{1+\frac{1}{4}\left[(1-\kappa)\left(1-e^{-i \beta}\right)\right.\right. \\
& \left.\left.\quad+(1+\kappa)\left(e^{i \beta}-1\right)\right]\right\}
\end{aligned}
$$

The parameter $k$ of the high-order upwind-biased differencing regulates the upwindedness: $\kappa=1$ yields central differencing, $\kappa=-1$ second-order accurate fully one-sided differencing, $k=1 / 3$ thirdorder accurate upwind-biased differencing. If a certain frequency $\beta$ needs to be perfectly damped, one inserts $z(\beta)$ into $P(z)$ and determines the values of $v$ and $\alpha$ that make the amplification factor vanish.

Applying a sequence of $m$ predictor-corrector methods generates schemes with an even number of stages $(2 m)$. To get a scheme with $2 m+1$ stages, a single application of the forward Euler time integration scheme

$$
u^{n+1}=(1+\lambda \Delta t) u^{n}
$$

should be added to the string of $m$ predictor-corrector methods. The forward-Euler scheme has amplification factor

$$
P_{1}(z)=1+z
$$

This polynomial has one zero at $z=-1$. For first-order upwind differencing, the spatial frequency that can be perfectly damped is $\beta=\pi$, and the required CFL number is $1 / 2$.

A general $\ell$-stage scheme with final marching time step $\Delta t$ can be written as

$$
\begin{gathered}
u^{(0)}=u^{n} \\
u^{(k)}=u^{n}+\gamma_{k} \lambda \Delta t u^{(k-1)}, \quad k=1, \ldots, \ell \\
u^{n+1}=u^{(\ell)}
\end{gathered}
$$

where $\gamma_{k}$ is the $k$ th coefficient of the $\ell$-stage scheme, and $\gamma_{\ell}=1$. The scheme has the amplification factor

$$
P_{\ell}(z)=1+z\left[1+\gamma_{\ell-1} z\left(1+\gamma_{\ell-2} z\left\{\cdots\left[1+\gamma_{2} z\left(1+\gamma_{1} z\right)\right] \cdots\right\}\right)\right]
$$

In the optimization procedure of Van Leer-Tai-Powell, a string of predictor-corrector methods, which damp different Fourier modes in the high-frequency range $[\pi / 2, \pi]$ and, possibly, a single forwardEuler step, are merged into the multistage scheme. Therefore, the amplification factor of the scheme can be written as

$$
\begin{gathered}
P_{2 m}(z)=\prod_{k=1}^{m}\left(1+z+\alpha_{k} z^{2}\right) \\
P_{2 m+1}(z)=(1+z) \prod_{k=1}^{m}\left(1+z+\alpha_{k} z^{2}\right)
\end{gathered}
$$

The coefficients and CFL number of the multistage scheme can be found by multiplying out and comparing Eqs. (11) and (12). But this combined multistage scheme may not be an optimal smoother. The optimization method of $\mathrm{Tai}^{3}$ and Van Leer et al. ${ }^{4}$ can now be implemented to minimize the maximum of the amplification factor in the high-frequency range. A set of perfectly damped frequencies is perturbed by means of Newton's iterative method, until all local maxima of the amplification factor in the high-frequency range are equalized within a specified margin. The parameters of optimized schemes for certain spatial discretizations, without use of residual smoothing, are shown in Table 1 . In the table, $\nu$ is the CFL number, $\gamma_{k}$ the $k$ th coefficient of the optimal multistage scheme, and $|P|_{\max }$ the equalized minimal maximum of the amplification factor of $\ell$-stage scheme in the range $[\pi / 2, \pi]$. The optimized schemes designed in this paper are for the first-order upwind scheme and a range of kappa schemes (excluding central differencing $\kappa=1$ ). From the table it is observed that the higher is the number of stages, the greater is the CFL number and the better is the damping of high-frequency errors.

The contours of the amplification factor of the five-stage scheme optimized for the third-order spatial differencing, $\kappa=1 / 3$, together with the locus of the Fourier transform $z(\beta)$ of the spatial operator (dashed line), are indicated in Fig. 1a. The contour levels for the magnitude of the amplification factor are $|P|=1,0.9,0.8,0.7,0.6$, $0.5,0.4,0.3,0.2,0.1,0.05$, and 0.01 . A graphic representations of the amplification factor as a function of $\beta$ is given in Fig. 1b for five different multistage schemes, all optimized for third-order differencing without residual smoothing. Note that the CFL number achieved in an $\ell$-stage scheme is considerably lower than the maximum stable CFL number for that $\ell$-stage scheme, which makes the optimal multistage schemes robust marching schemes.

\section{B. Modified Procedure}

The residual smoothing, using central differencing, is applied implicitly in the following form

$$
\left(1-\varepsilon \delta_{x}^{2}\right) \vec{R}=R
$$

where $R$ is the residual, $\bar{R}$ is the averaged residual, $\varepsilon$ is the smoothing coefficient, and $\delta_{x}^{2}$ is the discrete Laplace operator. Smoothing is applied in each stage. The Fourier transforms of the averaged residuals corresponding to Eqs. (6) and (7) are

$$
z(\beta)=\frac{-v\left(1-e^{-i \beta}\right)}{1+2 \varepsilon-\varepsilon\left(e^{i \beta}+e^{-i \beta}\right)}
$$

and

$$
\begin{aligned}
& z(\beta)= \\
& \frac{-\nu\left(1-e^{-i \beta}\right)\left\{1+\frac{1}{4}\left[(1-\kappa)\left(1-e^{-i \beta}\right)+(1+\kappa)\left(e^{i \beta}-1\right)\right]\right\}}{1+2 \varepsilon-\varepsilon\left(e^{i \beta}+e^{-i \beta}\right)}
\end{aligned}
$$

Using the optimization procedure of Sec. II.A, four-stage schemes for the third-order spatial differencing, with various smoothing coefficients, were obtained and are defined in Table 2. The damping and stability properties of these schemes are indicated in Figs. 2a and $2 \mathrm{~b}$. Although the maximum of the amplification factor in the high-frequency range has been minimized for each scheme, the magnitude of the amplification factor in the low-frequency range lies 
Table 1 Optimally smoothing multistage schemes for given spatial differencing without residual smoothing

\begin{tabular}{|c|c|c|c|c|c|c|c|c|}
\hline Stage & $v$ & $\gamma_{1}$ & $\gamma_{2}$ & $\gamma_{3}$ & $\gamma_{4}$ & $\gamma_{5}$ & $\gamma_{6}$ & $|P|_{\max }$ \\
\hline \multicolumn{9}{|c|}{ First-order upwind scheme } \\
\hline 2 . & 1.0 & 0.3333 & 1 & & & & & 0.3333 \\
\hline 3 & 1.5 & 0.1481 & 0.4000 & 1 & & & & 0.1414 \\
\hline 4 & 2.0 & 0.0834 & 0.2071 & 0.4267 & 1 & & & 0.0594 \\
\hline 5 & 2.5 & 0.0533 & 0.1263 & 0.2375 & 0.4414 & 1 & & 0.0244 \\
\hline 6 & 3.0 & 0.0370 & 0.0851 & 0.1521 & 0.2562 & 0.4512 & 1 & 0.0100 \\
\hline \multicolumn{9}{|c|}{ Kappa scheme, $\kappa=\frac{2}{3}$} \\
\hline 2 & 0.9134 & 1.1283 & 1 & & 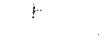 & & & 0.8094 \\
\hline 3 & 2.0336 & 0.3557 & 0.3739 & 1 & & & & 0.6527 \\
\hline 4 & 2.3240 & 0.2380 & 0.2850 & 0.5629 & 1 & & & 0.4304 \\
\hline 5 & 3.0437 & 0.1464 & 0.1876 & 0.3293 & 0.4895 & 1 & & 0.3034 \\
\hline 6 & 3.5851 & 0.1038 & 0.1415 & 0.2303 & 0.3227 & 0.5129 & 1 & 0.2077 \\
\hline \multicolumn{9}{|c|}{ Kappa scheme, $\kappa=\frac{1}{3}$} \\
\hline 2 & 0.8277 & 0.6612 & 1 & & & & & 0.7016 \\
\hline 3 & 1.3256 & 0.2883 & 0.5009 & 1 & & & & 0.4672 \\
\hline 4 & 1.7316 & 0.1668 & 0.3028 & 0.5276 & 1 & & & 0.2952 \\
\hline 5 & 2.1666 & 0.1067 & 0.1978 & 0.3233 & 0.5201 & 1 & & 0.1849 \\
\hline 6 & 2.5981 & 0.0742 & 0.1393 & 0.2198 & 0.3301 & 0.5178 & 1 & 0.1153 \\
\hline \multicolumn{9}{|c|}{ Kappa scheme, $\kappa=0$} \\
\hline 2 & 0.7029 & 0.5409 & 1 & & & & & 0.6633 \\
\hline 3 & 1.0559 & 0.2469 & 0.5210 & 1 & & & & 0.4210 \\
\hline 4 & 1.3999 & 0.1400 & 0.2939 & 0.5252 & 1 & & & 0.2576 \\
\hline 5 & 1.7484 & 0.0897 & 0.1866 & 0.3152 & 0.5216 & 1 & & 0.1559 \\
\hline 6 & 2.0975 & 0.0623 & 0.1288 & 0.2106 & 0.3251 & 0.5185 & 1 & 0.0938 \\
\hline \multicolumn{9}{|c|}{ Kappa scheme, $\kappa=-\frac{1}{3}$} \\
\hline 2 & 0.6054 & 0.4824 & 1 & & & & & 0.6429 \\
\hline 3 & 0.8949 & 0.2209 & 0.5149 & 1 & & & & 0.4006 \\
\hline 4 & 1.1884 & 0.1249 & 0.2809 & 0.5193 & 1 & & & 0.2411 \\
\hline 5 & 1.4841 & 0.0800 & 0.1757 & 0.3057 & 0.5174 & 1 & & 0.1433 \\
\hline 6 & 1.7805 & 0.0556 & 0.1201 & 0.2018 & 0.3178 & 0.5150 & 1 & 0.0848 \\
\hline \multicolumn{9}{|c|}{ Kappa scheme, $\kappa=-\frac{2}{3}$} \\
\hline 2 & 0.5294 & 0.4475 & 1 & & & & & 0.6288 \\
\hline 3 & 0.7808 & 0.2038 & 0.5038 & 1 & & & & 0.3884 \\
\hline 4 & 1.0370 & 0.1151 & 0.2694 & 0.5121 & 1 & & & 0.2314 \\
\hline 5 & 1.2951 & 0.0738 & 0.1670 & 0.2971 & 0.5120 & 1 & & 0.1361 \\
\hline 6 & 1.5537 & 0.0512 & 0.1135 & 0.1944 & 0.3109 & 0.5106 & 1 & 0.0797 \\
\hline \multicolumn{9}{|c|}{ Kappa scheme, $\kappa=-1$} \\
\hline 2 & 0.4693 & 0.4243 & 1 & & & & & 0.6178 \\
\hline 3 & 0.6936 & 0.1919 & 0.4930 & 1 & & & & 0.3799 \\
\hline 4 & 0.9214 & 0.1084 & 0.2601 & 0.5051 & 1 & & & 0.2248 \\
\hline 5 & 1.1507 & 0.0694 & 0.1603 & 0.2898 & 0.5067 & 1 & & 0.1313 \\
\hline 6 & $1: 3806$ & 0.0482 & 0.1085 & 0.1884 & 0.3049 & 0.5062 & 1 & 0.0763 \\
\hline
\end{tabular}

Table 2 Four-stage schemes modified by the Van Leer-Tai-Powell optimization without the modified procedure, for the third-order kappa scheme with IRS

\begin{tabular}{rcccccc}
\hline$\varepsilon$ & $\nu$ & $\gamma_{1}$ & $\gamma_{2}$ & $\gamma_{3}$ & $\gamma_{4}$ & \\
\hline 0 & 1.7316 & 0.1668 & 0.3028 & 0.5276 & 1 & Stable \\
0.1 & 2.2858 & 0.1712 & 0.2926 & 0.5168 & 1 & Stable \\
0.2 & 2.8179 & 0.1755 & 0.2868 & 0.5134 & 1 & Stable \\
0.3 & 3.3331 & 0.1795 & 0.2830 & 0.5129 & 1 & Stable \\
0.4 & 3.8367 & 0.1830 & 0.2802 & 0.5136 & 1 & Unstable \\
0.5 & 4.3325 & 0.1861 & 0.2781 & 0.5148 & 1 & Unstable \\
0.6 & 4.8227 & 0.1888 & 0.2764 & 0.5162 & 1 & Unstable \\
\hline \hline
\end{tabular}

outside the stability region, especially for the smoothing coefficients greater than 0.3 . It is seen that the instability becomes worse when increasing the smoothing coefficient in the IRS method. When using the Van Leer-Tai-Powell optimization, the resulting multistage schemes for all given spatial discretizations show the same unstable behavior. Clearly, the procedure has to be modified.
On the other hand, it is observed that the multistage coefficients obtained by applying the Van Leer-Tai-Powell optimization do not change significantly while the smoothing coefficients increase, even for $\varepsilon=0.5$ and $\varepsilon=0.6$, see Table 2 . This observation motivated the following modified procedure: keep the coefficients from the Van Leer-Tai-Powell optimization without residual smoothing, then search for the optimal CFL number that minimizes the area under the high-frequency amplification-factor curve while satisfying the stability condition for all low frequencies. After the modified procedure, the CFL number for the first-order upwind scheme and the kappa schemes $(\kappa=1 / 3,-1)$ with various smoothing coefficients become those listed in Table 3 . It is seen that the CFL number now increases with increasing smoothing coefficient. For instance, the three-stage scheme obtained by the Van LeerTai-Powell optimization for the third-order kappa scheme without residual smoothing has a CFL number of 1.3256 , as seen in Table 1. Applying the modified procedure, a scheme results with a CFL number of 3.0029. Figure 3a shows the amplification factor for the two optimal schemes and also for the smoothed scheme using the optimal CFL number of nonsmoothed scheme. 


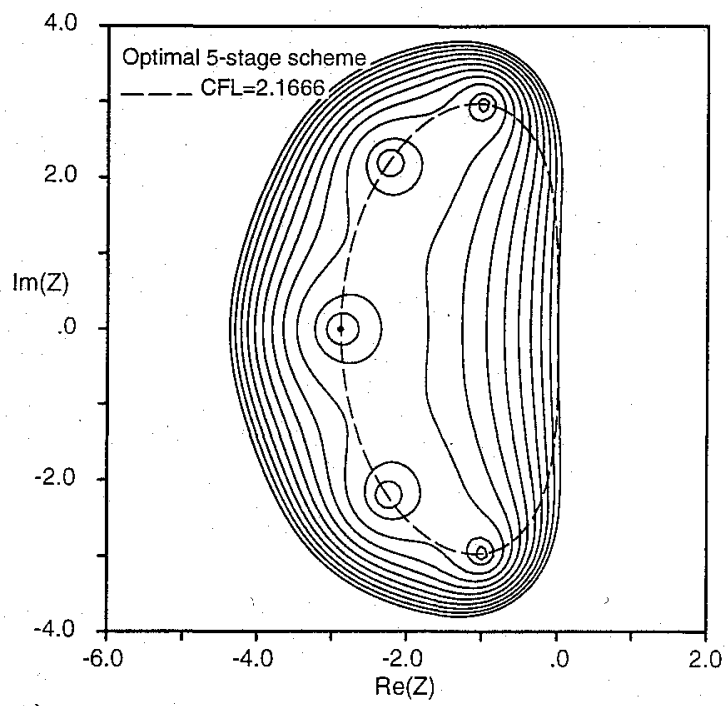

a)

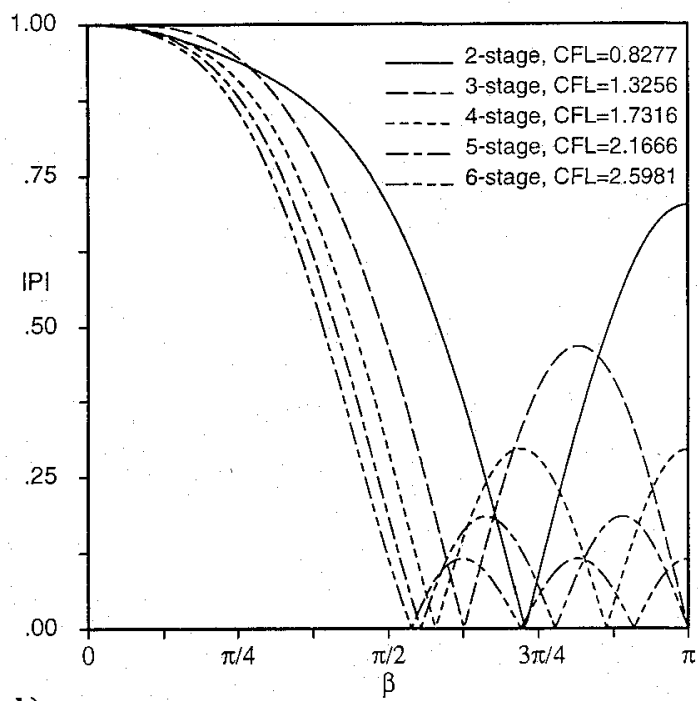

b)

Fig. 1. Optimal multistage schemes, for the third-order scheme without residual smoothing: a) locus and contours of optimal five-stage scheme and b) amplification factor.

Residual smoothing can also be implemented explicitly (ERS), to suit parallel computing and unstructured grids. In this case the residual $R$ is replaced by the average

$$
\bar{R}=\left(1+\varepsilon \delta_{x}^{2}\right) R
$$

at each stage of multistage scheme. Using central differencing in the ERS method, the complex function $z(\beta)$ corresponding to Eqs. (6) and (7) become

$$
z(\beta)=-v\left(1-e^{-i \beta}\right)\left[1-2 \varepsilon+\varepsilon\left(e^{i \beta}+e^{-i \beta}\right)\right]
$$

and

$$
\begin{aligned}
& z(\beta)=-\nu\left(1-e^{-i \beta}\right)\left\{1+\frac{1}{4}\left[(1-\kappa)\left(1-e^{-i \beta}\right)\right.\right. \\
& \left.\left.+(1+\kappa)\left(e^{i \beta}-1\right)\right]\right\}\left[1-2 \varepsilon+\varepsilon\left(e^{i \beta}+e^{-i \beta}\right)\right]
\end{aligned}
$$

It is seen that for $\varepsilon \geq 1 / 4$, there are Fourier modes such that $\vec{R}$ could be zero when $R$ is not. Applying the modified procedure, the CFL number for the first-order upwind scheme and the kappa schemes $(\kappa=-1,1 / 3)$ with various smoothing coefficient were obtained and are listed in Table 4 . It is seen that the CFL number increases with increasing smoothing coefficient. For instance, the three-stage scheme obtained by the Van Leer-Tai-Powell optimization for the third-order scheme without residual smoothing has a CFL number of 1.3256 , as seen in Table 1. Applying the modified procedure for $\varepsilon=0.2$, a scheme results with a CFL number of

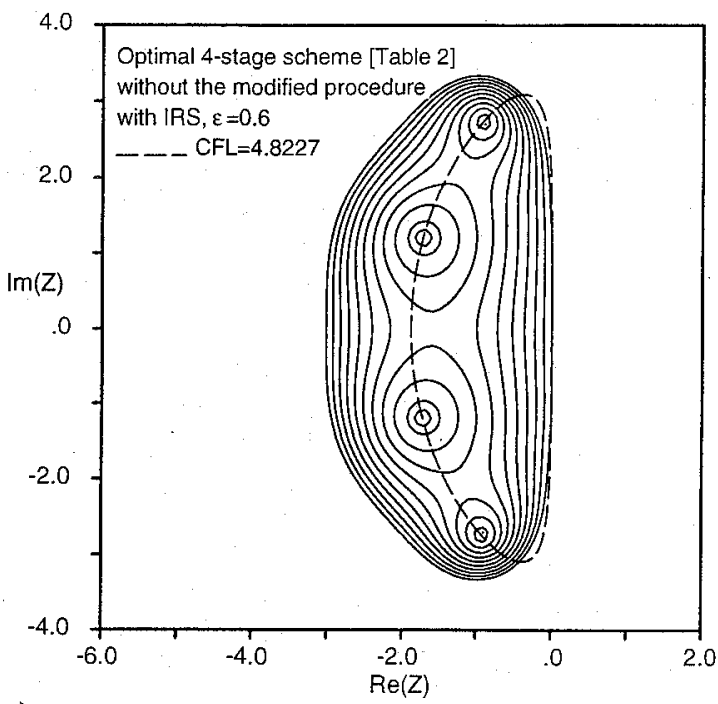

a)

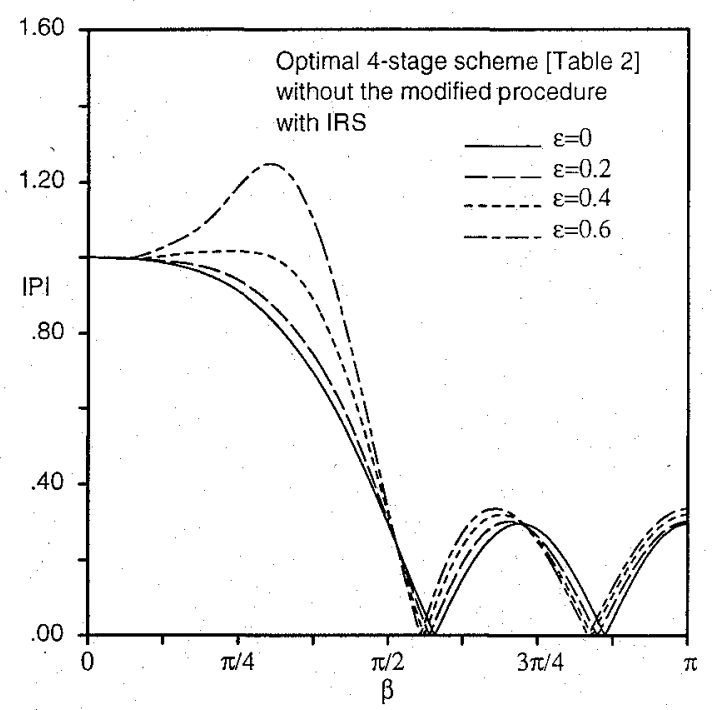

b)

Fig. 2. Optimal four-stage scheme without the modified procedure, for the third-order scheme with IRS: a) locus and contours and b) amplification factor.

2.3180. Figure $3 b$ shows the amplification factor for the two optimal schemes and also for the smoothed scheme using the optimal CFL number of nonsmoothed scheme. From Tables 3 and 4, it is observed that the CFL number for the ERS method is larger than one for the IRS method with the same smoothing coefficient. It is seen that the damping and stability are greatly improved by the modified procedure. Thus, the procedure makes the schemes a good "smoother" as multigrid strategy requires.

\section{Extension to Multidimensional Problem}

Applying the multistage schemes optimized by one-dimensional analysis to multidimensional or axisymmetric convection problems is not so straightward. Fortunately, as shown by Tai, ${ }^{3}$ a twodimensional minimax technique for a two-dimensional convection leads to optimal multistage coefficients that are not significantly different from those obtained by one-dimensional optimization, although the optimal CFL number may differ somewhat. Thus, the practical strategy proposed in this paper is keeping the coefficients from one-dimensional analysis and redefining a two-dimensional CFL number.

It is obvious that the schemes should be optimal with regard to errors propagating in the convection direction; this only requires extending the definition of Courant number to the case of convection through a two-dimensional grid. Consider a two-dimensional cell $\mathrm{ABCD}$, as shown in Fig. 4; set the convection speed in the $x$ and $y$ directions equal to $\boldsymbol{a}$ and $\boldsymbol{b}$. From a consideration of numerical 


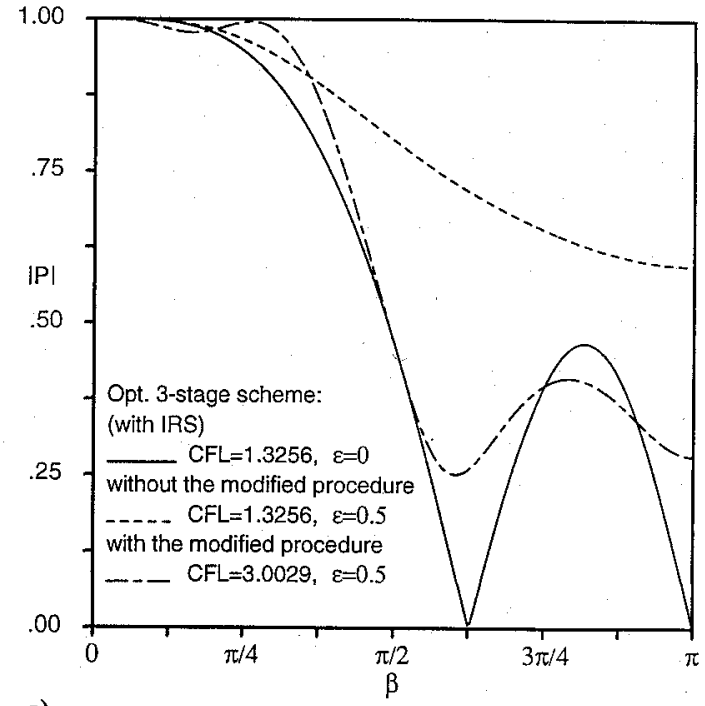

a)

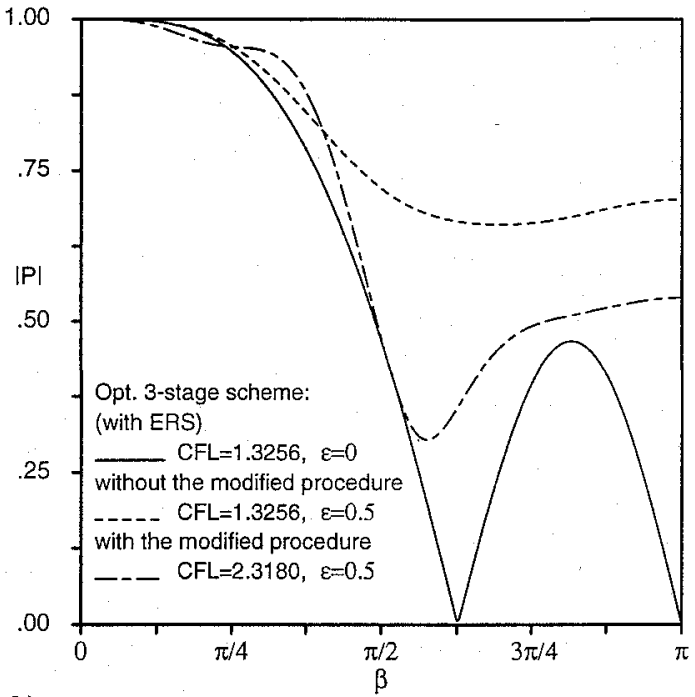

b)

Fig. 3 Amplification factor of optimal three-stage scheme with and without the modified procedure for the third-order scheme with a) IRS and b) ERS.

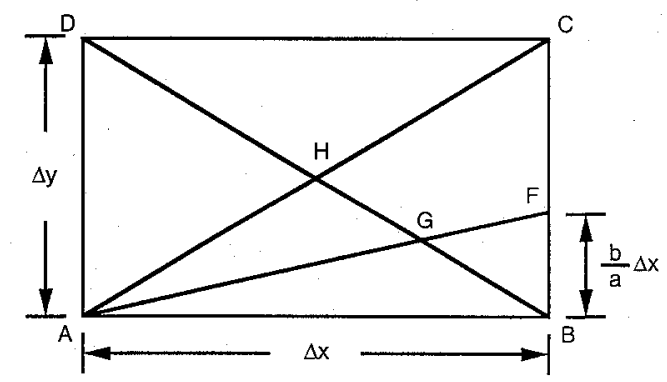

Fig. 4 Geometry of two-dimensional cell.

stability, the characteristic cell width in the convection direction should not be greater than $\overline{\mathrm{AG}}$. From geometric relations, the length of $\overline{\mathrm{AG}}$ can be found to be

$$
|\overline{\mathrm{AG}}|=\frac{\Delta x \Delta y}{b \Delta x+a \Delta y} \sqrt{a^{2}+b^{2}}
$$

and the CFL number based on this length would be redefined as

$$
v=\frac{\Delta t \sqrt{a^{2}+b^{2}}}{\overline{\mathrm{AG}}}=\Delta t\left(\frac{|a|}{\Delta x}+\frac{|b|}{\Delta y}\right)
$$

where $v$ is the CFL number obtained from one-dimensional analysis. This may not be the perfect choice, but it has been shown to be very
Table 3 CFL number of optimally smoothing multistage scheme for

\begin{tabular}{|c|c|c|c|c|c|}
\hline $\begin{array}{l}\text { Stage } \\
\varepsilon\end{array}$ & 2 & 3 & 4 & 5 & 6 \\
\hline \multicolumn{6}{|c|}{ First-order upwind scheme } \\
\hline 0.1 & 1.2847 & 1.9054 & 2.5876 & 3.2888 & 3.9952 \\
\hline 0.2 & 1.5720 & 2.2673 & 3.0182 & 3.7879 & 4.5891 \\
\hline 0.3 & 1.8443 & 2.6233 & 3.4262 & 4.2299 & 5.0704 \\
\hline 0.4 & 2.1023 & 2.9687 & 3.8403 & 4.6909 & 5.5719 \\
\hline 0.5 & 2.3435 & 3.2888 & 4.2336 & 5.1381 & 6.0448 \\
\hline 0.6 & 2.5699 & 3.5856 & 4.5889 & 5.5370 & 6.4675 \\
\hline 0.7 & 2.7832 & 3.8630 & 4.9178 & 5.9035 & 6.8637 \\
\hline 0.8 & 2.9835 & 4.1207 & 5.2250 & 6.2483 & 7.2427 \\
\hline 0.9 & 3.0038 & 4.3682 & 5.5173 & 6.5784 & 7.6017 \\
\hline 1.0 & 3.0120 & 4.6020 & 5.7940 & 6.8902 & 7.9502 \\
\hline \multicolumn{6}{|c|}{ Kappa scheme, $\kappa=\frac{1}{3}$} \\
\hline 0.1 & 1.1014 & 1.6683 & 2.1633 & 2.6925 & 3.2306 \\
\hline 0.2 & 1.2038 & 1.9907 & 2.5748 & 3.1804 & 3.7854 \\
\hline 0.3 & 1.3730 & 2.2804 & 2.9509 & 3.6430 & 4.3233 \\
\hline 0.4 & 1.5267 & 2.5419 & 3.2887 & 4.0564 & 4.8088 \\
\hline 0.5 & 1.6555 & 2.7804 & 3.5971 & 4.4317 & 5.2747 \\
\hline 0.6 & 1.7414 & 3.0029 & 3.8827 & 4.7799 & 5.6526 \\
\hline 0.7 & 1.8225 & 3.1562 & 4.1478 & 5.1041 & 6.0342 \\
\hline 0.8 & 1.8996 & 3.2842 & 4.4008 & 5.4127 & 6.3954 \\
\hline 0.9 & 1.9734 & 3.4052 & 4.6401 & 5.7046 & 6.7395 \\
\hline 1.0 & 2.0442 & 3.5206 & 4.8695 & 5.9862 & 7.0690 \\
\hline \multicolumn{6}{|c|}{ Kappa scheme, $\kappa=-1$} \\
\hline 0.1 & 0.2552 & 0.8267 & 1.1674 & 1.4851 & 1.8082 \\
\hline 0.2 & 0.2562 & 0.8303 & 1.4002 & 1.7725 & 2.1619 \\
\hline 0.3 & 0.2572 & 0.8338 & 1.6205 & 2.0321 & 2.4654 \\
\hline 0.4 & 0.2581 & 0.8374 & 1.8354 & 2.2808 & 2.7446 \\
\hline 0.5 & 0.2592 & 0.8409 & 2.0493 & 2.5258 & 3.0219 \\
\hline 0.6 & 0.2601 & 0.8439 & 2.2615 & 2.7701 & 3.2964 \\
\hline 0.7 & 0.2611 & 0.8471 & 2.4741 & 3.0145 & 3.5691 \\
\hline 0.8 & 0.2621 & 0.8503 & 2.6859 & 3.2579 & 3.8421 \\
\hline 0.9 & 0.2630 & 0.8536 & 2.8929 & 3.5040 & 4.1180 \\
\hline 1.0 & 0.2640 & 0.8567 & 3.0956 & 3.7487 & 4.4016 \\
\hline
\end{tabular}
IRS with various smoothing coefficient

robust in the sense of reproducing the good damping properties of the optimized one-dimensional schemes for two-dimensional convection.

\section{Fast Euler Solver}

A fast Euler solver was developed to prove the applicability of the optimally smoothing multistage schemes to an axisymmetric Euler flow problem. An axisymmetric secant-ogive-cylinder-boattail projectile (SOCBT) is used as the testing model. The compressible inviscid external flow of the projectile is described by the Euler equations in conservation form. A finite volume method was chosen, i.e., the integratial formulation of the equations is directly discretized in physical space. A system of ordinary differential equations in time is obtained by applying the spatial discretization in each cell. First-order upwind differencing and the Van Leer kappa schemes were chosen to be the spatial discretization. Roe's flux-difference splitting, ${ }^{12}$ modified to satisfy the entropy condition, ${ }^{13}$ was adopted to evaluate the fluxes through the control surfaces. The Van Albada limiter was imposed on the kappa schemes to prevent numerical oscillations.

The residual smoothing is applied on the finest and coarser grid levels. In the two-dimensional case, the implicit and explicit steps are applied in product forms

$$
\left(1-\varepsilon_{x} \delta_{x}^{2}\right)\left(1-\varepsilon_{y} \delta_{y}^{2}\right) \bar{R}=R
$$

and

$$
\bar{R}=\left(1+\varepsilon_{x} \delta_{x}^{2}\right)\left(1+\varepsilon_{y} \delta_{y}^{2}\right) R
$$

i.e., approximation factorization is applied to obtain the averaged residual. It is not at all clear that a two-dimension analysis for $\varepsilon_{x}=\varepsilon_{y}$ would yield an optimal CFL number that is 
Table 4 CFL number of optimally smoothing multistage scheme for ERS with various smoothing coefficients

\begin{tabular}{|c|c|c|c|c|c|}
\hline \multicolumn{6}{|l|}{ Stage } \\
\hline$\varepsilon$ & 2 & 3 & 4 & 5 & 6 \\
\hline \multicolumn{6}{|c|}{ First-order upwind scheme } \\
\hline 0.05 & 1.1643 & 1.7448 & 2.3780 & 3.0165 & 3.7033 \\
\hline 0.10 & 1.4150 & 2.0323 & 2.7130 & 3.4498 & 4.1951 \\
\hline 0.15 & 1.7580 & 2.4101 & 3.1089 & 3.8613 & 4.6450 \\
\hline 0.20 & 2.1048 & 2.8921 & 3.6673 & 4.4714 & 5.3020 \\
\hline 0.25 & 2.2368 & 3.1791 & 4.0907 & 5.0179 & 5.9530 \\
\hline \multicolumn{6}{|c|}{ Kappa scheme, $k=\frac{1}{3}$} \\
\hline 0.05 & 0.9277 & 1.5233 & 1.9799 & 2.4739 & 2.9075 \\
\hline 0.10 & 1.0862 & 1.7878 & 2.3019 & 2.8420 & 3.3047 \\
\hline 0.15 & 1.2512 & 2.0744 & 2.6707 & 3.2877 & 3.8016 \\
\hline 0.20 & 1.3784 & 2.3180 & 3.0014 & 3.7031 & 4.2938 \\
\hline 0.25 & 1.4018 & 2.4442 & 3.2158 & 3.9956 & 4.6588 \\
\hline \multicolumn{6}{|c|}{ Kappa scheme, $\kappa=-1$} \\
\hline 0.05 & 0.2547 & 0.7920 & 1.0638 & 1.3525 & 1.6435 \\
\hline 0.10 & 0.2552 & 0.9608 & 1.2510 & 1.5912 & 1.9447 \\
\hline 0.15 & 0.2557 & 1.1725 & 1.4781 & 1.8377 & 2.2250 \\
\hline 0.20 & 0.2562 & 1.1936 & 1.8010 & 2.1802 & 2.5854 \\
\hline 0.25 & 0.2567 & 1.1949 & 2.1868 & 2.6208 & 3.0573 \\
\hline
\end{tabular}

close to the value derived from one-dimension analysis. However, we have already redefined a two-dimensional CFL number suited for two-dimensional convection term. It has been shown that using the redefined CFL number will not make the optimal multistage schemes unstable. Therefore, the smoothing coefficients used in the different directions are set to be equal in this paper.

Recall that the multigrid method effectively removes the lowfrequency components of error on the coarser grids in the iterative procedure but requires a good single-grid smoother. Thus, the use of a multistage scheme with good high-frequency damping on each grid may effectively improve the performance of the multigrid strategy. ${ }^{2,14}$ Therefore, the optimally smoothing multistage scheme was considered as the time-integration method. The multigrid strategy used in the test code is a saw-tooth cycle, ${ }^{2}$ in which first-order upwind differencing is used on all coarse grids and the kappa schemes only on the finest grid. A powerful technique is to perform some preliminary iterations on a coarser grid, then use the resulting approximation as an initial guess on the finer grid; this so-called "nested iteration" was used in the fastsolver to get a better initial quess on the finest grid. In addition, one more acceleration technique was used, i.e., local time stepping which allows each cell to advance in time at maximum rate and makes the scalar, constant-coefficient analysis locally applicable.

\section{Application of the Optimally Smoothing Multistage Schemes}

The numerical application of the optimal multistage schemes was carried out for the SOCBT projectile in supersonic flow, $M_{\infty}=1.2$, at zero angle of attack. All computations were performed on an HP-750 workstation and achieved a drop of residual to $10^{-6}$. The computational work required for convergence, denoted as WU, is expressed in terms of finest-grid residual calculations. Most of the test cases were performed by using a $64 \times 64$ mesh which was generated by O-type elliptic solver. Figure 5 is the expanded view of the grid.

\section{A. Grid Analysis}

The quality of the solution and the effect of mesh size on convergence can be inferred from Figs. 6-8. Figure 6 shows the pressure coefficient [defined as $C_{p}=\left(p-p_{\infty}\right) / 1 / 2 \rho_{\infty} U_{\infty}^{2}$ ] on the projectile, obtained by using the optimal five-stage scheme for the first-order upwind scheme with the IRS method, $\varepsilon=0.5$, on singleand five-level grid calculations $(64 \times 64$ cells $)$. The surface pres-

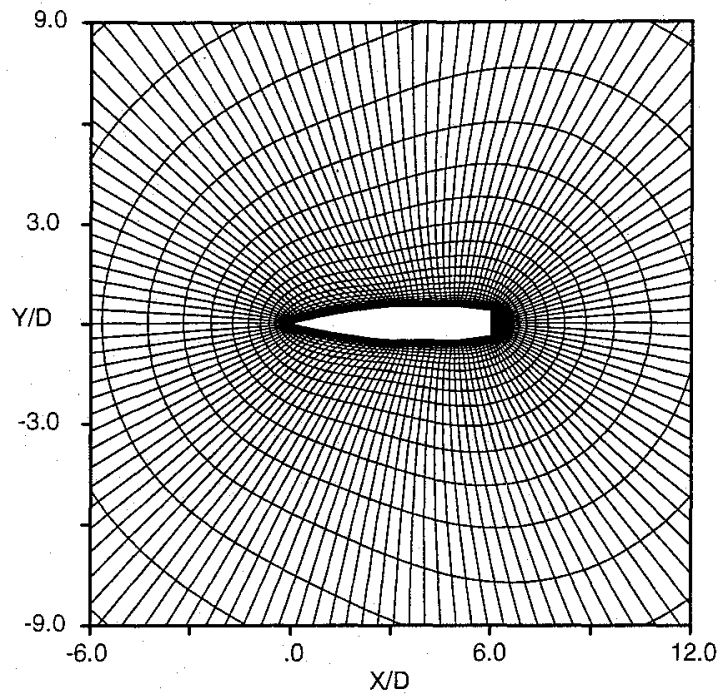

Fig. 5 Expanded view of computational grid near the model.

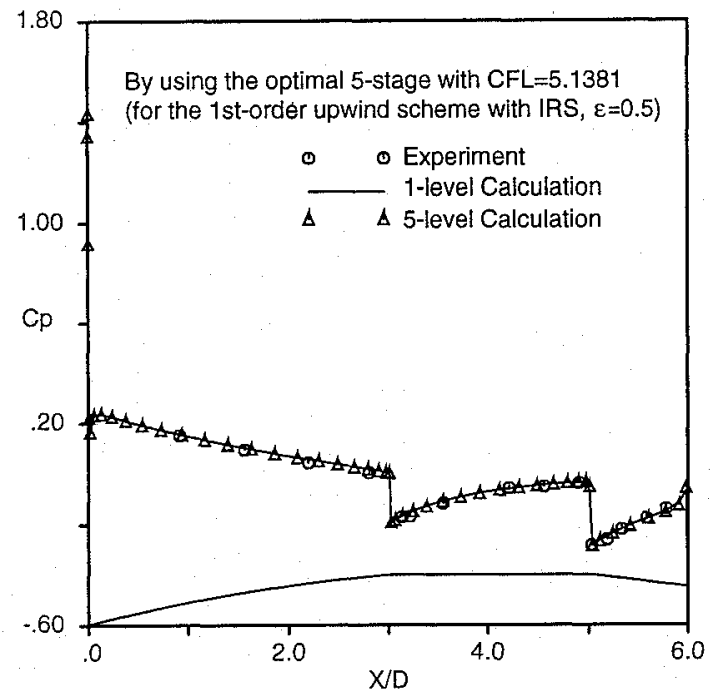

Fig. 6 Surface pressure distribution of the test model.

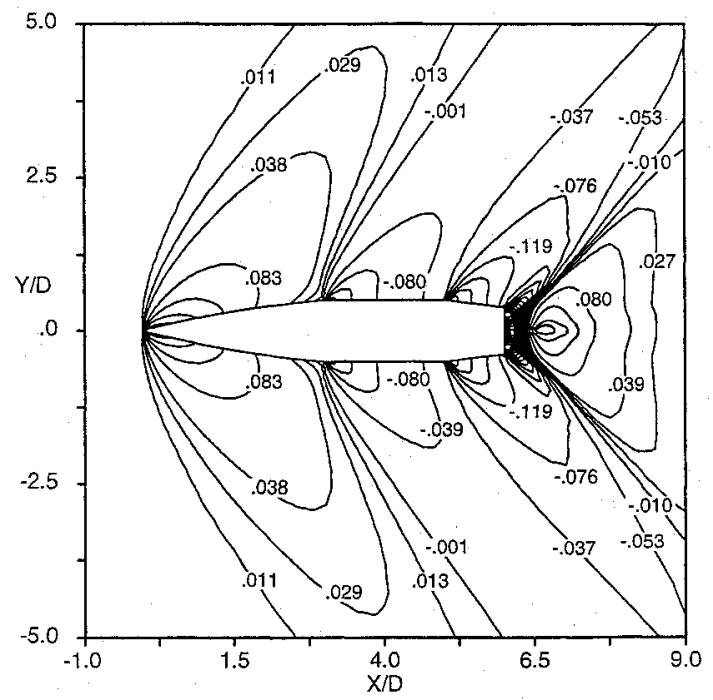

Fig. 7 Pressure contours of the test model.

sure distributions of the SOCBT projectile for both calculations are identical and in agreement with the experimental data. ${ }^{15}$ Figure 7 is the pressure contour plot for the projectile at $M_{\infty}=1.2$. In this figure, the flow pattern of projectile, such as an attached shock, expansions, and a recompression shock can be found. Meshes with different numbers of cells, $64 \times 64,128 \times 64$, and $128 \times 128$, were 


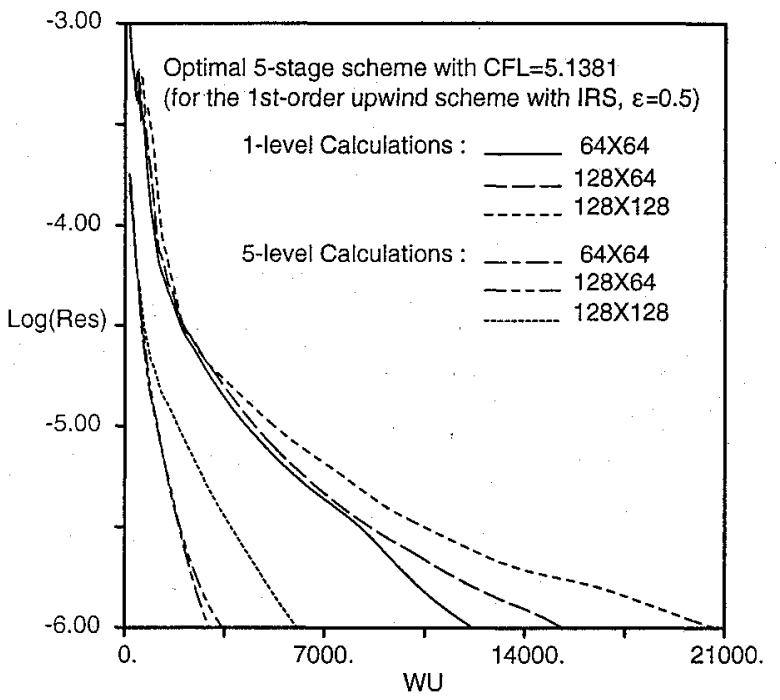

Fig. 8 Convergence history of optimal five-stage scheme on single- and five-level grids with different grid size.

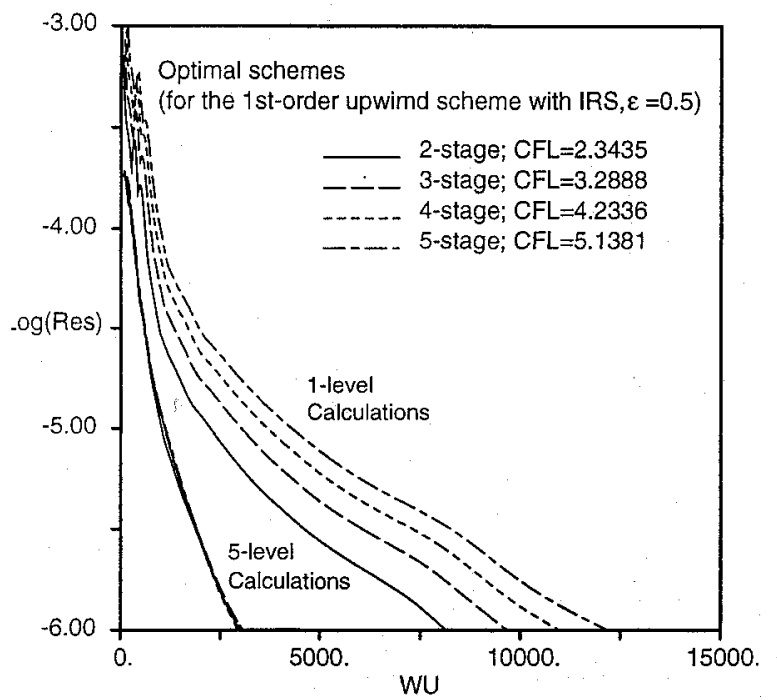

Fig. 9 Convergence history of optimal schemes with different number of stage.

used to test convergence dependence on cell size. Figure 8 shows results of the optimal five-stage scheme for the first-order upwind scheme with the IRS method, $\varepsilon=0.5$, implemented on a singleand a five-level grid. It is seen that the multigrid convergence is not completely independent of the number of cells in the finest grid.

\section{B. Covergence Effect of the Optimal Multistage Stages}

The effect of the number of stages on convergence can be seen in Fig. 9. The results in this figure are obtained by using the optimal two-, three-, four- and five-stage schemes for the first-order upwind scheme with IRS, $\varepsilon=0.5$, on single- and five-level grids. It is shown that the convergence of these schemes is effectively enhanced by using the multigrid strategy. The convergence of the two-stage scheme is superior to the others for a. single-level calculation. This is because it uses fewer flux calculations per unit CFL number. However, for the five-level calculations, the convergence histories of all of the schemes are similar. This is because in multigrid relaxation the damping properties become important, and these are better for the many-stage schemes. The effect of the number of grid levels can be seen in Fig. 10, showing the convergence histories of calculation for various numbers of levels, by using for the optimal five-stage scheme and the first-order upwind scheme with the IRS and $\varepsilon=0$. The convergence history of the single-grid calculation illustrates that initially the residual decreases sharply, corresponding to the quick elimination of the high-frequency modes,

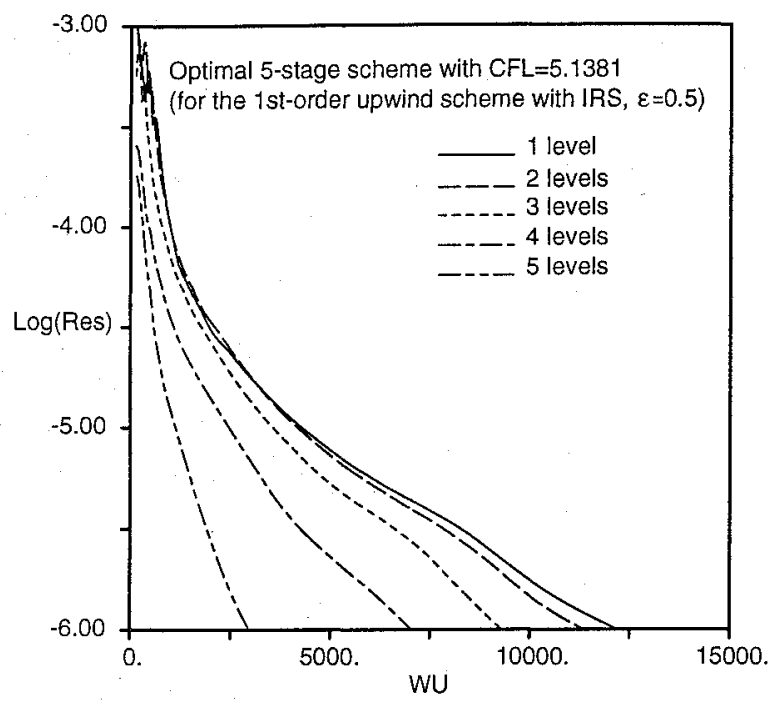

Fig. 10 Convergence history of optimal scheme on grids with different number of levels, first-order upwind scheme.

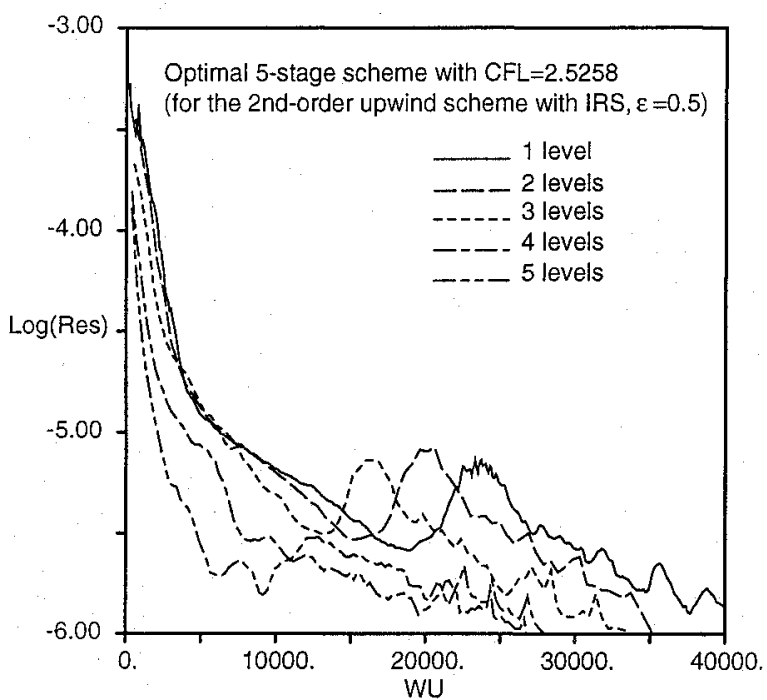

Fig. 11 Convergence history of optimal scheme on grids with different number of levels, second-order upwind scheme.

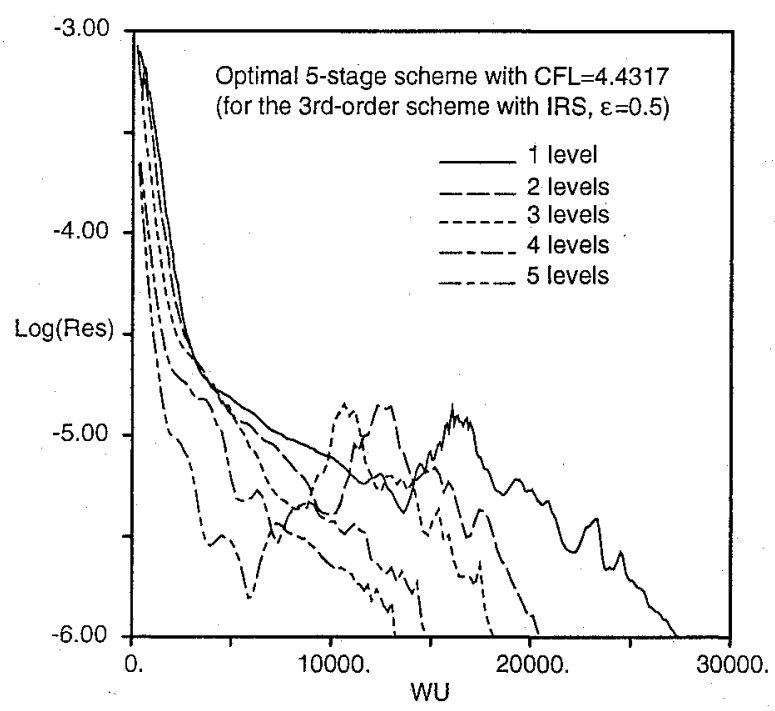

Fig. 12 Convergence history of optimal scheme on grids with different number of levels, third-order scheme.

followed by a slow decrease due to the low-frequency modes. The five-level calculation displays the best convergence performance, illustrating that the multigrid code based on the optimal scheme effectively benefits from the damping of the low-frequency errors. Figures 11 and 12 show the multilevel convergence behavior of 


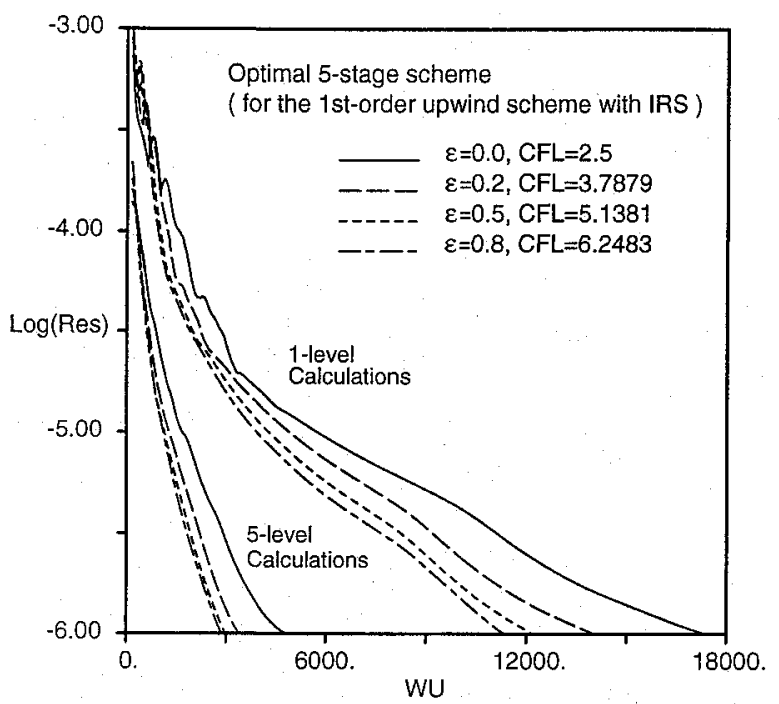

Fig. 13 Convergence history of optimal scheme with different smoothing coefficients, IRS.

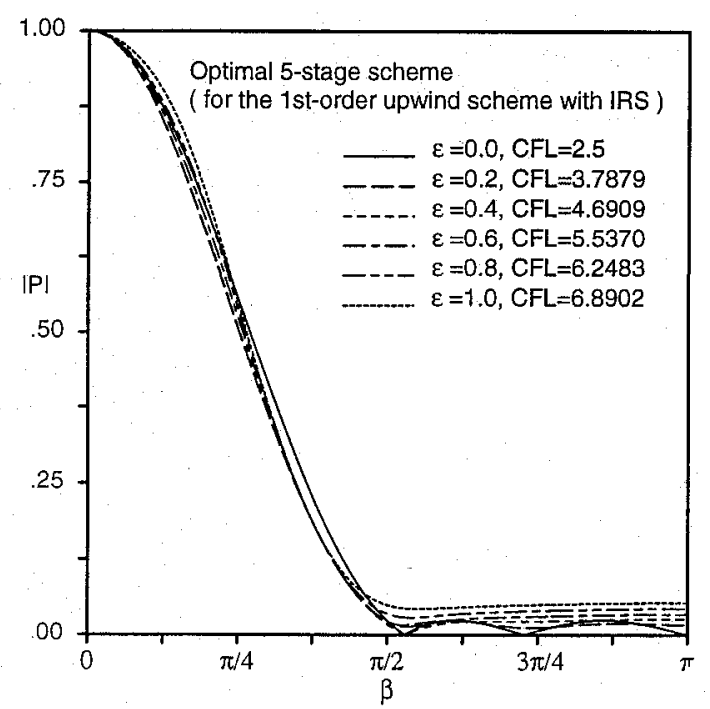

Fig.14 Amplification factor of optimal five-stage scheme with different smoothing coefficients, IRS.

optimal five-stage schemes for the kappa scheme $(\kappa=-1,1 / 3)$ with the IRS method, $\varepsilon=0.5$.

A convergence comparison of the optimal five-stage schemes for first-order upwind differencing with IRS, $\varepsilon=0,0.2,0.5$, and 0.8 , on single- and five-level calculations is shown in Fig. 13. It is seen that the speed of convergence increases while the smoothing coefficient increases, as expected, because of the better damping properties and the larger Courant number. It is also seen that the convergence efficiency does not improve dramatically by increasing the smoothing coefficient beyond about 0.5 . Figure 14 shows that the high-frequency damping properties worsen while the smoothing coefficient increases. This also holds for the kappa schemes.

Figure 15 shows the convergence histories of the optimal fivestage schemes for first-order differencing with ERS, $\varepsilon=0,0.1$, and 0.2 on single- and five-level calculations. It is seen that the five-level calculation with $\varepsilon=0.2$ yields the best convergence efficiency. From Fig. 13 and 15, it is seen that the ERS method yields better convergence performance while using the same smoothing coefficient. In our experiment, using the ERS method with $\varepsilon \geq 1 / 4$ may make the calculation diverge, unless a very small CFL number is employed.

\section{Comparison with the Runge-Kutta Scheme}

Two cases with five-stage Runge-Kutta schemes, one with the CFL number 2.5 and one with the allowable maximum CFL

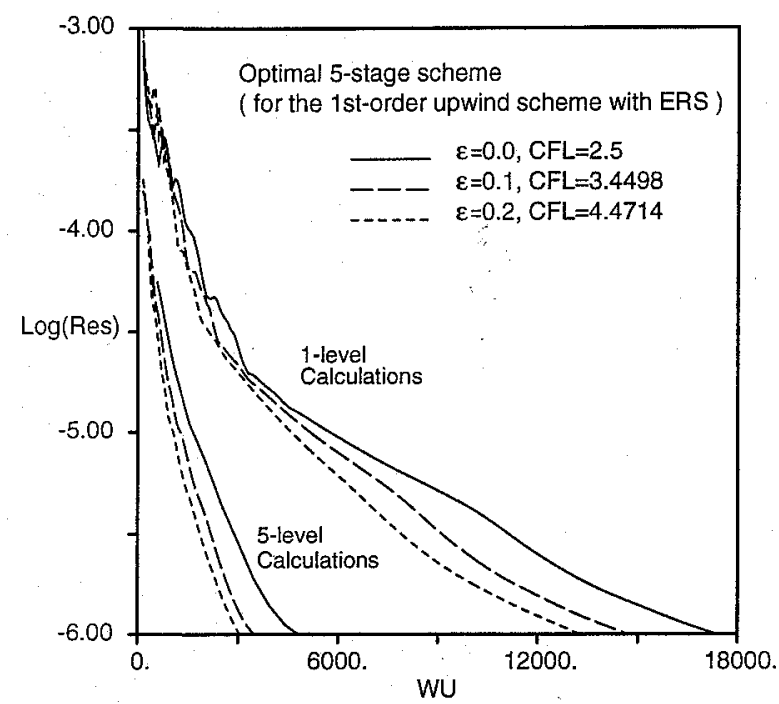

Fig. 15 Convergence history of optimal scheme with different smoothing coefficients, ERS.

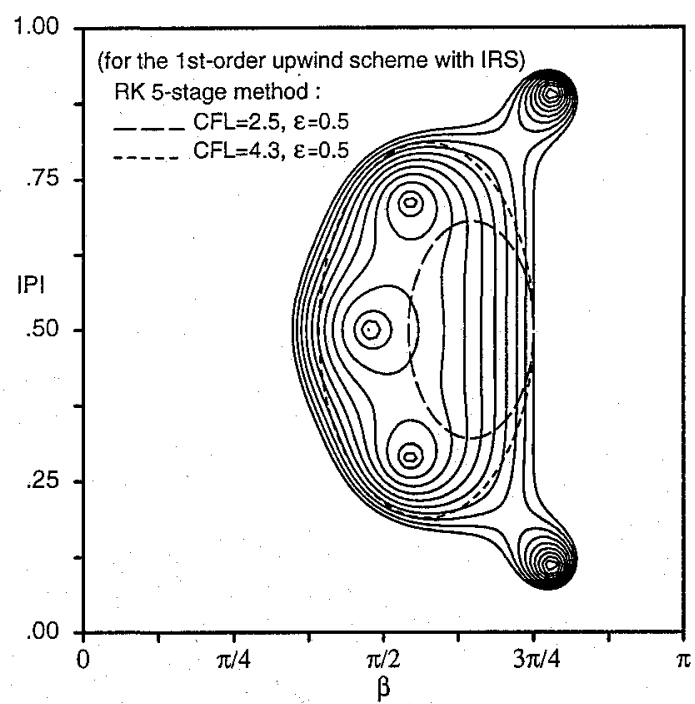

Fig. 16a Loci and contours of Runge-Kutta five-stage scheme.

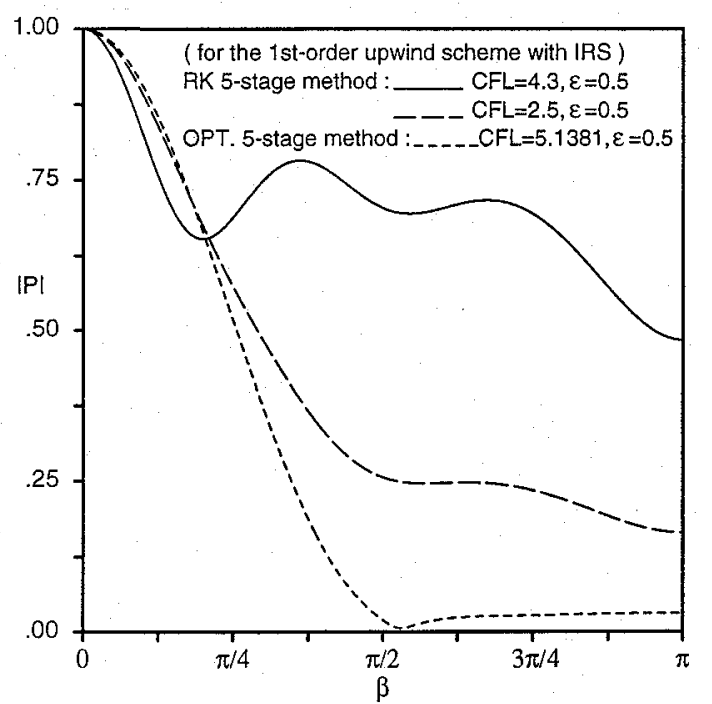

Fig. 16b Amplification factor of optimal and Runge-Kutta five-stage schemes.

number 4.3 , were used to compare with the optimal five-stage scheme. The implicit residual smoothing, such that $\varepsilon=0.5$, is considered in these schemes. The damping and stability of the RungeKutta four-stage scheme can be judged from Figs. 16a and 16b. The comparisons were implemented both on a single- and a fivelevel grid by utilizing the five-stage schemes for first-order upwind 


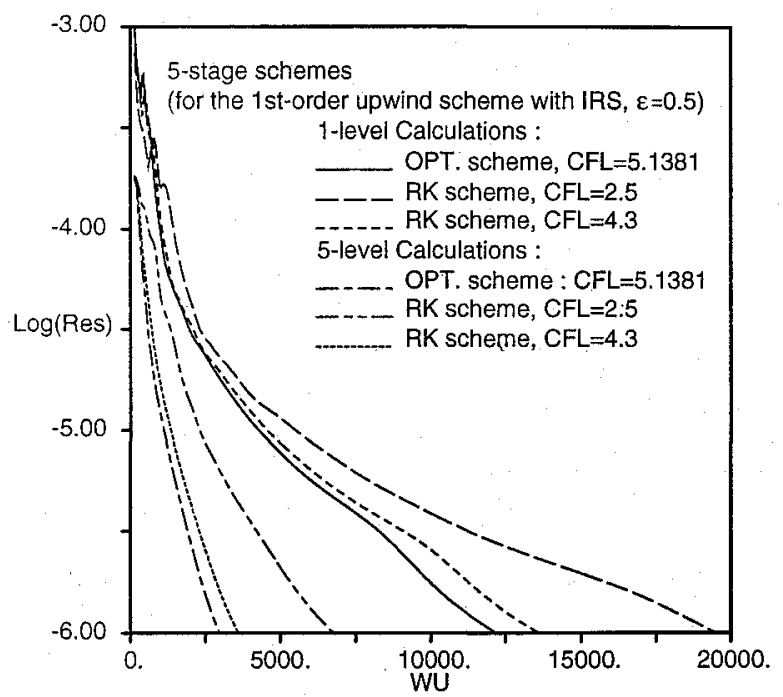

Fig. 17 Comparison of convergence history of optimal five-stage scheme and five-stage Runge-Kutta scheme.

differencing with the IRS method, $\varepsilon=0.5$, as shown in Fig. 17. The optimal scheme performs somewhat better than the Runge-Kutta scheme, presumably because of its large CFL number.

\section{Conclusions}

In this paper, a technique has been introduced for developing a multistage scheme suited for multigrid use. Parameters for multistage schemes for certain one-dimensional spatial discretizations, with or without residual smoothing, implicit or explicit, are presented. The damping and stability properties of some optimally smoothing multistage schemes are studied. The extension to any specified spatial discretization with or without residual smoothing should be easily achieved with little computational cost. The multistage coefficients from one-dimensional analysis directly apply to multidimensional computations by redefining the CFL number. In numerical applications of the optimally smoothing schemes this significantly accelerates the convergence to a steady-state solution.

\section{Acknowledgment}

The authors gratefully acknowledge the financial support of the National Science Council of the Republic of China under Grant NSC-82-0401-E-014-009.

\section{References}

${ }^{1}$ Jameson, A., Schmidt, W., and Turkel, E., "Numerical Solutions of the Euler Equations by a Finite Volume Method Using Runge-Kutta Timestepping Schemes," AIAA Paper 81-1259, June 1981.

${ }^{2}$ Jameson, A., "Numerical Solution of the Euler Equations for Compressible Inviscid Fluids," Numerical Methods for the Euler Equations of Fluid Dynamics, edited by Angrand, F., Dervieux, A., Desideri, J. A., and Glowinski, R., Society for Industrial and Applied Mathematics, Philadelphia, PA, 1985, pp. 199-245.

${ }^{3}$ Tai, C. H., "Acceleration Techniques for Explicit Euler Codes," Ph.D. Thesis, Univ. of Michigan, Ann Arbor, MI, May 1990.

${ }^{4}$ Van Leer, B., Tai, C. H., and Powell, K. H., "Design of Optimally Smoothing Multi-stage Scheme for the Euler Equations," AIAA Paper 89. 1923-CP, June 1989.

${ }^{5}$ Catalano, L. A., and Deconinck, H., "Two Dimensional Optimization of Multistage Schemes Applied to Hyperbolic Equations," von Kármán Institute for Fluid Dynamics, TN-173, Rhode Saint Genese, Belgium, July 1990.

${ }^{6}$ Lynn, J. F., and Van Leer, B., "Multistage Schemes for the Euler and Navier-Stokes Equations with Optimal Smoothing," AIAA Paper 93-3355, June 1993.

${ }^{7}$ Van Leer, B., Lee, W. T., Roe, P. L., Powell, K. G., and Tai, C. H., "Design of Optimally Smoothing Multi-stage Scheme for the Euler Equations," Communications in Applied Numerical Methods, Vol. 8, 1992, pp. 761-769.

${ }^{8}$ Enander, R., "A New Residual Smoother," Uppsala Univ., Dept. of Scientific Computing, Rept. No. 128, 1990.

${ }^{9}$ Blazek, L., Kroll, N., Radespiel, R., and Rossow, C.-C., "Upwind Implicit Residual Smoothing Method for Multi-stage Scheme," AIAA Paper 91-1532, June 1991

${ }^{10}$ Van Leer, B., "Upwind-difference Methods for Aerodynamic Problems Governed by the Euler Equations," Lectures in Applied Mathematics, Vol. 22,1985 , pp. $327-336$.

${ }^{11}$ Hirsch, C., "Numerical Computation of Internal and External Flow," Ist ed., Vol. 1, Wiley, New York, 1988, pp. 237-261.

${ }_{12}$ Roe, P. L., "Approximate Riemann Solver, Parameter Vector and Difference Scheme," Journal of Computational Physics, Vol. 43, 1981, pp. $357-372$.

${ }^{13}$ Van Leer, B., Lee, W. T., and Powell, K. G., "Sonic-Point Capturing," AIAA Paper 89-1945, June 1989.

${ }^{14}$ Briggs, W. L., A Multigrid Tutorial, Society for Industrial and Applied Mathematics, Philadelphia, PA, 1987.

${ }^{15}$ Kayser, L. D., and Whiton, F., "Surface Pressure Measurements on Boatailed Projectile Shape at Transonic Speeds," U.S. Army Ballistic Research Lab., Aberdeen Proving Ground, MD, AD-A113520, March 1982. 